\title{
Predictors of poor health-related quality of life among hemodialysis patients with anemia in Jordan
}

\author{
Osama Y. Alshogran ${ }^{1 *} \mathbb{0}$, Esraa A. Shatnawi ${ }^{1}$ Shoroq M. Altawalbeh ${ }^{1}$, Anan S. Jarab ${ }^{1}$ and Randa I. Farah ${ }^{2}$
}

\begin{abstract}
Objective: This study examined health-related quality of life (HRQOL) and factors associated with poor HRQoL among hemodialysis (HD) patients.

Methods: A multicenter cross-sectional study was conducted on HD patients with anemia in Jordan $(n=168)$. Validated questionnaires were utilized to collect data on HRQoL using EQ-5D-5L, psychiatric symptoms using Hospital Anxiety and Depression Scale (HADS), and comorbidities score using the modified Charlson Comorbidity Index $(\mathrm{mCCl})$. Multiple linear regression analysis was conducted to identify the variables which are independently associated with HRQoL among patients.
\end{abstract}

Results: The mean $( \pm \mathrm{SD}$ ) age of study participants was 52.2 ( \pm 14.6 ) years. The mean utility value of EQ-5D-5L was 0.44 ( \pm 0.42$)$. Participants reported extreme problems mostly in pain/discomfort domain (19.6\%). Increased age, increased $\mathrm{mCCl}$ and patient complains, more years under dialysis, decreased exercise, and low family income were significantly associated with poor HRQoL $(p<0.05)$.

Conclusion: The study findings revealed poor HRQoL among HD patients with anemia. Various dimensions of health were negatively affected among HD patients. Development and implementation of appropriate approaches with adequate education and psychosocial support to HD patients by healthcare professionals targeting improved HRQOL and clinical outcomes would be necessary.

Keywords: Hemodialysis, Quality of life, EQ-5D-5L, Anemia, Predictors

\section{Introduction}

Chronic kidney disease (CKD) is a common global health problem. End-stage renal disease (ESRD) represents the ultimate progressive stage of CKD where patients are primarily dependent on hemodialysis (HD) as part of renal replacement therapy [1]. Patients with ESRD have multiple co-morbid conditions including diabetes and hypertension, and are more prone to renal-related complications such as imbalance in calcium and phosphate

*Correspondence: oyalshogran@just.edu.jo

${ }^{1}$ Department of Clinical Pharmacy, Faculty of Pharmacy, Jordan University of Science and Technology, Irbid 22110, Jordan

Full list of author information is available at the end of the article homeostasis, vitamin D deficiency, anemia, and secondary hyperparathyroidism [2-4], which together may significantly impact patients' well-being and health outcomes. Additionally, maintenance dialysis by itself is an invasive procedure which subjects patients to additional pain and discomfort [5]. Anemia is prevalent in CKD, especially in patients receiving dialysis [6, 7]. Iron supplementations and erythropoiesis-stimulating agents (ESA) are the main treatment modalities for anemia in HD patients [8].

Health-related quality of life (HRQoL) is considered a multi-dimensional ultimate health outcome measure which should be frequently monitored in patients with ESRD [9]. Several studies documented poor HRQoL 
among ESRD patients [10-13], with lower HRQoL being reported among CKD patients with anemia [14]. An earlier study showed that HD patients with anemia who received high ESA dosing or intravenous iron therapy had slightly better HRQoL scores after 1-3 months follow-up period [15]. Poor HRQoL was linked to increased hospitalization and mortality among ESRD patients [9, 16]. Therefore, one of the primary goals of ESRD management is to implement interventions that improve HRQoL. Compromised HRQoL among ESRD patients could be associated with physical, social, psychological and adherence factors $[17,18]$.

The primary treatment modality for ESRD patients in Jordan is HD. Based on the Jordan Renal Registry data, the total number of ESRD patients in Jordan in 2019 was 6708. Of them, 6316 patients were treated in dialysis units. A total of 6165 (97.6\%) were receiving HD, while only 151 patients (2.4\%) were undergoing peritoneal dialysis. The majority of patients in Jordan were males (63\%) with age of more than 40 years old (76\%). To date, limited information exists about variables associated with poor HRQoL among HD patients with anemia, particularly in Jordan. Thus, this study was conducted to evaluate HRQoL among a representative group of HD patients with anemia in Jordan, and the independent associations of multiple factors with poor HRQoL.

\section{Methods}

\section{Study design and setting}

This cross-sectional study was conducted on HD patients at different dialysis units in Jordan from January through October 2018. Patients who visit HD clinics at King Abdullah University Hospital (KAUH), Jordan University Hospital (JUH), Princess Basma Hospital, and AlRamtha Governmental Hospital were approached during their scheduled dialysis sessions. The inclusion criteria included HD patients older than 18 years who have been under dialysis for at least three months and able to communicate. After screening for eligibility, patients were informed about study objectives and invited to participate by a trained clinical pharmacist. Patients were assured about the right to withdraw and that the collected information will be kept confidential. All participants signed a written informed consent. Patient's interview was conducted after 30-60 min of the start of dialysis, with an average interview time of 15-20 min.

\section{Sociodemographics and clinical data}

Socio-demographics including age, gender, weight and height, material status, educational level, employment, family status, family income per month, and smoking status were collected. Medical files were also used to collect information on cause of ESRD, dialysis access, time since first dialysis, dialysis sessions per week, length of dialysis session, current medications, and patient major complains. Biochemical data of hemoglobin ( $\mathrm{Hgb})$, ferritin, creatinine, urea, calcium, potassium, parathyroid hormone, albumin, were also obtained from medical reports.

\section{Study instruments}

The primary outcome that was measured in the present study was subject's HRQoL and was assessed using the generic EQ-5D-5L instrument developed by Euro QOL Group. Participants were asked to complete an Arabic version of the EQ-5D-5L questionnaire which was obtained from the developer with permission (ID: 21432). The first part of the instrument is a descriptive system of patient's HRQoL in terms of five dimensions of health including mobility, self-care, usual activities, pain/discomfort, and anxiety/depression. The answers for each dimension were rated on a five-level scale of no problems, slight problems, moderate problems, severe problems and unable to/extreme problems. The collected responses were then scored to calculate the index value using the value sets (weights) from United Kingdom general population scoring algorithm (i.e. EQ-5D-5L Crosswalk Index Value Calculator) [19]. The index value for each individual could range from -0.594 to 1 . Higher index values indicate better quality of life and vice versa.

The second part of the instrument is the EQ Visual Analogue Scale (EQ_VAS) which was used to evaluate patients' self-rated health states on a rating scale ranging from 0 to 100 , with higher scores indicating better quality of life level [20].

The EQ-5D is a reliable scale that has been validated in Arabic language [21, 22]. The instrument has been widely used among different disease conditions in Jordan [23, $24]$ and was implemented previously among HD patients $[14,25]$.

A previously validated and highly-implemented Arabic version of the hospital anxiety and depression scale (HADS) was utilized to explore psychiatric symptoms among study participants [26, 27]. This instrument contains seven items for each subscale of anxiety and depression, with scores ranging between 0 and 21 for each subscale. High scores indicate more psychiatric distress. The modified Charlson comorbidity index (mCCI) in ESRD which includes 18 comorbidities was used to calculate patients' comorbidity score as previously described [28]. Each disease condition was assigned a specific weight that ranges between 1 and 10. For example, dementia was given a weight of 1 while the presence of metastatic disease was given a weight of 10 [28]. The score for each patient was generated according to his/ her comorbidities, then the mean score of the study sample was calculated. Higher scores indicate higher 
comorbidity burden. Additionally, information about major patients complains that might be related to disease or medications as well as knowledge about indication of prescribed medications were collected from participants.

The questionnaire was initially developed by the research investigators based on previous literature as described earlier. A pilot study was then conducted among a group of HD patients $(n=10)$ to ensure that the questionnaire items are understandable and clear to study subjects. The developed survey was generally clear to the pilot subjects with few recommendations to rephrase some of questions and make the survey a little shorter. The questionnaire items were then updated based on patients' suggestions. The questionnaire was retested among a couple of patients to ensure clarity. Data of the pilot study were not included in the final analysis.

\section{Statistical analysis}

Patient' demographics and clinical characteristics were presented using simple descriptive statistics. Categorical data were listed as counts and percentages while continuous data were reported as arithmetic means with standard deviation. Multivariable linear regression was conducted to identify variables associated with poor HRQoL. Variables (sociodemographic, clinical, medical, labs, etc.) included in regression models were selected using backward stepwise process with a $P<0.2$ to stay. Data analyses were conducted using STATA version 14 (StataCorp. 2015. Stata. Statistical Software: Release 14. College Station, TX: StataCorp LP.)

\section{Results}

Patients socio-demographics and clinical characteristics

A total of 245 patients were approached and invited to participate. Eleven subjects were excluded as they did not match the criteria and 66 patients refused to participate. Accordingly, a total of 168 patients were interviewed by the research pharmacist and entered to the final analysis, giving a response rate of $71.8 \%$. More than half of the patients $(59.5 \%)$ were male. Participants mean $( \pm S D)$ age was $52.2( \pm 14.6)$ years, and ranged from 18 to 85 years. A quarter of the patients were obese (25\%), half were current smoker or ex-smoker (50.6\%), and the majority were married (66.7\%). The majority of participants were unemployed (85.7\%) and had up to school education level (72.6\%). Other socio-demographic characteristics are listed in Table 1.

Regarding subjects' clinical and medical characteristics (Table 2), self-reported diabetes was the main etiology for ESRD in the study (32.9\%) followed by hypertension $(30.4 \%)$. The majority of the patients (89\%) were prescribed iron therapy including oral formulation such as
Table 1 Socio-demographic features of the studied population

\begin{tabular}{|c|c|}
\hline Variable & Number (\%) N=168 \\
\hline \multicolumn{2}{|l|}{ Age (years) } \\
\hline $18-40$ & $35(20.8)$ \\
\hline $40-64$ & $97(57.7)$ \\
\hline$>65$ & $36(21.4)$ \\
\hline \multicolumn{2}{|l|}{ Gender } \\
\hline Male & $100(59.5)$ \\
\hline Female & $68(40.5)$ \\
\hline \multicolumn{2}{|l|}{$B M I\left(\mathrm{~kg} / \mathrm{m}^{2}\right)$} \\
\hline Underweight $<18.5$ & $9(5.4)$ \\
\hline Normal range 18.5-24.9 & $73(43.5)$ \\
\hline Overweight 25-29.9 & $44(26.2)$ \\
\hline Obese $>30$ & $42(25.0)$ \\
\hline \multicolumn{2}{|l|}{ Marital status } \\
\hline Single & $34(20.2)$ \\
\hline Married & $112(66.7)$ \\
\hline Divorced or widow & $22(13.1)$ \\
\hline \multicolumn{2}{|l|}{ Education level } \\
\hline Illiterate & $10(5.9)$ \\
\hline Junior school & $50(29.8)$ \\
\hline High school & $62(36.9)$ \\
\hline College or over & $46(27.4)$ \\
\hline \multicolumn{2}{|l|}{ Employment status } \\
\hline Employed & $24(14.3)$ \\
\hline Unemployed & $144(85.7)$ \\
\hline \multicolumn{2}{|l|}{ Family status } \\
\hline Live alone & $8(4.8)$ \\
\hline Live with partner & $15(8.9)$ \\
\hline Live with family & $137(81.6)$ \\
\hline Others & $8(4.8)$ \\
\hline \multicolumn{2}{|l|}{ Family income (month) } \\
\hline Low (less than 250 JD) & $52(30.9)$ \\
\hline Moderate (250-500 JD) & $83(49.4)$ \\
\hline High (more than 500 JD) & $33(19.7)$ \\
\hline \multicolumn{2}{|l|}{ Smoking status } \\
\hline Smoker & $50(29.8)$ \\
\hline Nonsmoker & $83(49.4)$ \\
\hline Ex-smoker & $35(20.8)$ \\
\hline \multicolumn{2}{|l|}{ Co-morbidities } \\
\hline Hypertension & $113(67.3)$ \\
\hline Diabetes mellitus & $68(40.9)$ \\
\hline
\end{tabular}

BMI, Body Mass Index

ferrous gluconate, ferrous sulphate, and iron complex or intravenous products as iron sucrose and iron dextran. Most of the patients (>75\%\%) were also prescribed erythropoietin, vitamin $\mathrm{D}$, and calcium carbonate. Only about half of them were aware about the indication for use of such medications $(47.4 \%$ for iron and $50.2 \%$ for erythropoietin). The majority of the patients $(90.5 \%)$ received 
Table 2 Patients clinical, medical and laboratory information

\begin{tabular}{|c|c|}
\hline Variable & $\mathrm{N}(\%)$ \\
\hline \multicolumn{2}{|l|}{ Cause of ESRD } \\
\hline HTN & $38(22.8)$ \\
\hline DM & $43(25.8)$ \\
\hline HTN and DM & $12(7.2)$ \\
\hline Glomerulonephritis & $5(2.9)$ \\
\hline Others & $2(1.2)$ \\
\hline Drugs & $1(0.6)$ \\
\hline Medical error & $66(39.3)$ \\
\hline Unknown & $69(41.7)$ \\
\hline \multicolumn{2}{|l|}{ Number of prescribed medications } \\
\hline$<5$ & $19(11.6)$ \\
\hline $5-10$ & $90(54.9)$ \\
\hline$>10$ & $55(33.5)$ \\
\hline \multicolumn{2}{|l|}{ Vascular access for HD } \\
\hline Catheter & $39(23.2)$ \\
\hline Fistula & $117(69.6)$ \\
\hline Graft & $12(7.2)$ \\
\hline \multicolumn{2}{|l|}{ Dialysis sessions per week } \\
\hline 2 Times weekly & $16(9.5)$ \\
\hline 3 Times weekly & $152(90.5)$ \\
\hline \multicolumn{2}{|l|}{ Length of dialysis session in hours } \\
\hline $3 \mathrm{~h}$ or less & $32(19.1)$ \\
\hline $3.5 \mathrm{~h}$ & $57(33.9)$ \\
\hline 4 h or more & $79(47.0)$ \\
\hline \multicolumn{2}{|l|}{ Years under dialysis } \\
\hline$<2$ years & $51(30.4)$ \\
\hline $2-5$ years & $52(30.9)$ \\
\hline$>5$ years & $65(38.7)$ \\
\hline Patients on iron products & $149(88.7)$ \\
\hline Patients on erythropoietin & $159(94.6)$ \\
\hline \multicolumn{2}{|l|}{ Psychiatric symptoms $^{a}$} \\
\hline HADS-A & $5.2( \pm 4.5)$ \\
\hline HADS-D & $6.4( \pm 4.9)$ \\
\hline HADS-T & $11.6( \pm 8.9)$ \\
\hline Patients had practiced exercise last week & $37(22.16)$ \\
\hline Duration of exercise per week in minutes ${ }^{\mathrm{a}}$ & $148.11( \pm 126.3)$ \\
\hline Number of emergency visits last year ${ }^{a}$ & $2.94( \pm 4.18)$ \\
\hline Number of hospital admissions last year ${ }^{\mathrm{a}}$ & $1.66( \pm 2.19)$ \\
\hline Serum creatinine ${ }^{a}$ (normal range $0.7-1.3 \mathrm{mg} / \mathrm{dL}$ ) & $9.22( \pm 2.69)$ \\
\hline Serum urea" (normal range: $2.5-7.1 \mathrm{mmol} / \mathrm{L}$ ) & $21.36( \pm 6.76)$ \\
\hline Calcium level $\mathrm{l}^{\mathrm{a}}$ (normal range $2.2-2.6 \mathrm{mmol} / \mathrm{L}$ ) & $2.18( \pm 0.23)$ \\
\hline Potassium level ${ }^{a}$ (normal range $3.5-5.0 \mathrm{mmol} / \mathrm{L}$ ) & $5.29( \pm 0.83)$ \\
\hline Parathyroid hormone (normal range 10-65 ng/L) & $633.7( \pm 626.8)$ \\
\hline Iron ${ }^{\mathrm{a}}$ (normal range $11-29 \mu \mathrm{mol} / \mathrm{L}$ ) & $9.43( \pm 11.46)$ \\
\hline Hemoglobin ${ }^{\mathrm{a}}$ (normal range male $14-17$, female $12-16 \mathrm{~g} / \mathrm{dL}$ ) & $10.27( \pm 1.59)$ \\
\hline Total iron binding capacity ${ }^{\mathrm{a}}$ (normal range $250-460 \mu \mathrm{g} / \mathrm{dL}$ ) & $233.97( \pm 45.47)$ \\
\hline Serum ferritina (normal range 15-200 ng/mL) & $458.58( \pm 640.36)$ \\
\hline
\end{tabular}

HTN, hypertension; DM, diabetes mellitus; HADS-A, hospital anxiety and depression scale-anxiety; HADS-D, hospital anxiety and depression scale-depression; HADS-T, hospital anxiety and depression scale-total

${ }^{a}$ Data are presented as mean $( \pm S D)$ 
dialysis three times a week, of which (69.6\%) were performed using fistula as a vascular access. Furthermore, most of patients (69.6\%) are on dialysis for more than two years. The mean $( \pm \mathrm{SD}) \mathrm{mCCI}$ score for participants was $4.22( \pm 3.62)$ which ranged between zero and 18 . The major comorbid conditions observed among our sample were diabetes $(40.5 \%)$ and peripheral vascular disease (32.1\%). The mean $( \pm \mathrm{SD}) \mathrm{Hgb}$ level was $10.27( \pm 1.59) \mathrm{g} /$ $\mathrm{dL}$, and the mean iron level was $9.43( \pm 11.46) \mu \mathrm{mol} / \mathrm{L}$. Other patient's laboratory data which were collected at the time of interview are shown in the Table 2.

About a quarter of patients (22.6\%) had severe gastrointestinal symptoms during the last week of interview. One-third (32.7\%) of patients reported severe joint and muscle pain and $32.7 \%$ of patients had severe insomnia. Overall, less than half of participants were aware about the indication of prescribed medications.

\section{Quality of life and health status}

The EQ-5D-5L results are shown in Fig. 1. The dimension that showed the highest response rate of "no problems" was "self-care" (63.1\%). While, the dimension with the lowest response rate for "no problems" was "pain/discomfort" (23.2\%). The mean utility or index score $( \pm S D)$ was $0.44( \pm 0.42)$, ranging from -0.594 to 1.0 . The number (\%) of patients who answered "extreme problem or unable to" for the five items of EQ-5D-5L was as follows: anxiety/depression 27 (16.07\%), pain/discomfort 33 (19.64\%), usual activities 24 (14.29\%), self-care $22(13.10 \%)$ and mobility 15 (8.93\%). The mean EQ-VAS $( \pm S D)$ value was $62.05 \pm 22.61$

The results of multivariate regression analysis revealed that increased age, longer years under dialysis, increased comorbidities (mCCI), and patients general complains were significantly and negatively associated with HRQoL, while increased income and doing exercise were positively associated with HRQoL (Table 3). For example, one unit increase in the mCCI score was significantly associated with a decrease in HRQoL index score by -0.026 .

\section{Discussion}

The current study investigated HRQoL and its associated variables in patients with HD in Jordan. Results clearly suggest compromised HRQoL among study participants with the worst health status for pain/discomfort and mobility dimensions. Longer years under dialysis, increased age, increased number of comorbid conditions and patients' major complaints were associated with poor HRQoL, while higher income and conducting exercise were associated with better HRQoL.

Although HRQoL among HD patients has been investigated at earlier time in Jordan $[18,29]$, the current study is the first one to use the EQ-5D-5L to explore HRQoL among HD patients in Jordan. The latter instrument is simple, easy to be completed, well recognized, requires shorter time to be finished, and has enhanced sensitiviy as compared with other available instruments for measuring HRQoL among patient population [13, 30]. We found that the mean index $( \pm S D)$ value among HD patients was 0.44 ( \pm 0.42$)$, while findings from other studies that used the same instrument in Palestine, Japan and Korea found utility values of $0.37( \pm 0.440)$ [13], $0.809( \pm 0.184)$ [31] and $0.704( \pm 0.199)$ [32] respectively. This variation in HRQoL could be related partially to differences in sociodemographic and clinical characteristics including age, HD duration, and the presence of comorbid conditions in the recruited sample.

An earlier study conducted in Jordan reported a moderately decreased level of HRQoL among ESRD patients using Quality of Life Index-Dialysis Version III (QLIDVIII) [18]. The latter study also revealed adherence to treatment, depression, and perceived seriousness of illness as significant predictors of HRQoL [18]. Another study used the Quality of Life Index showed that HD patients in Jordan had moderate to high life satisfaction [29]. Shdaifat et al. used the RAND 36-Item Health Survey in Jordan which showed that patients were found to have lower HRQoL when compared to their caregivers and to the general population [33].

Consistent with earlier research findings [13, 32], results in the present study showed that increased age was significantly associated with lower HRQoL. For example, Zyoud et al. [13] revealed that younger patients ( $<30$ years $)$ were found to have significantly better HRQoL than older one using the EQ-5D-5L instrument. This could be attributed partially to the lower physical activity and to the increased number of comorbid conditions in old patients [34].

In agreement with the findings of previous studies [12, $13,35,36]$, the current study revealed a negative association between comorbidity index and HRQoL. For instance, a study conducted among Singaporean patients with renal failure documented that low Charlson comorbidity index was significantly linked with better HRQoL [35]. This association could be explained by the burden of increased number of comorbid conditions as well as the need for a higher number of medications and their associated negative consequences on health.

In contrast to the present research findings, Yang et al. [35] reported similar or better mental health status among patients with longer dialysis vintage versus those with shorter dialysis vintage. Our finding of the assocaition between decreased quality of life with longer dialysis years, could be attributed to increased economic burden and dialysis complications with longer years under dialysis. The observation that 


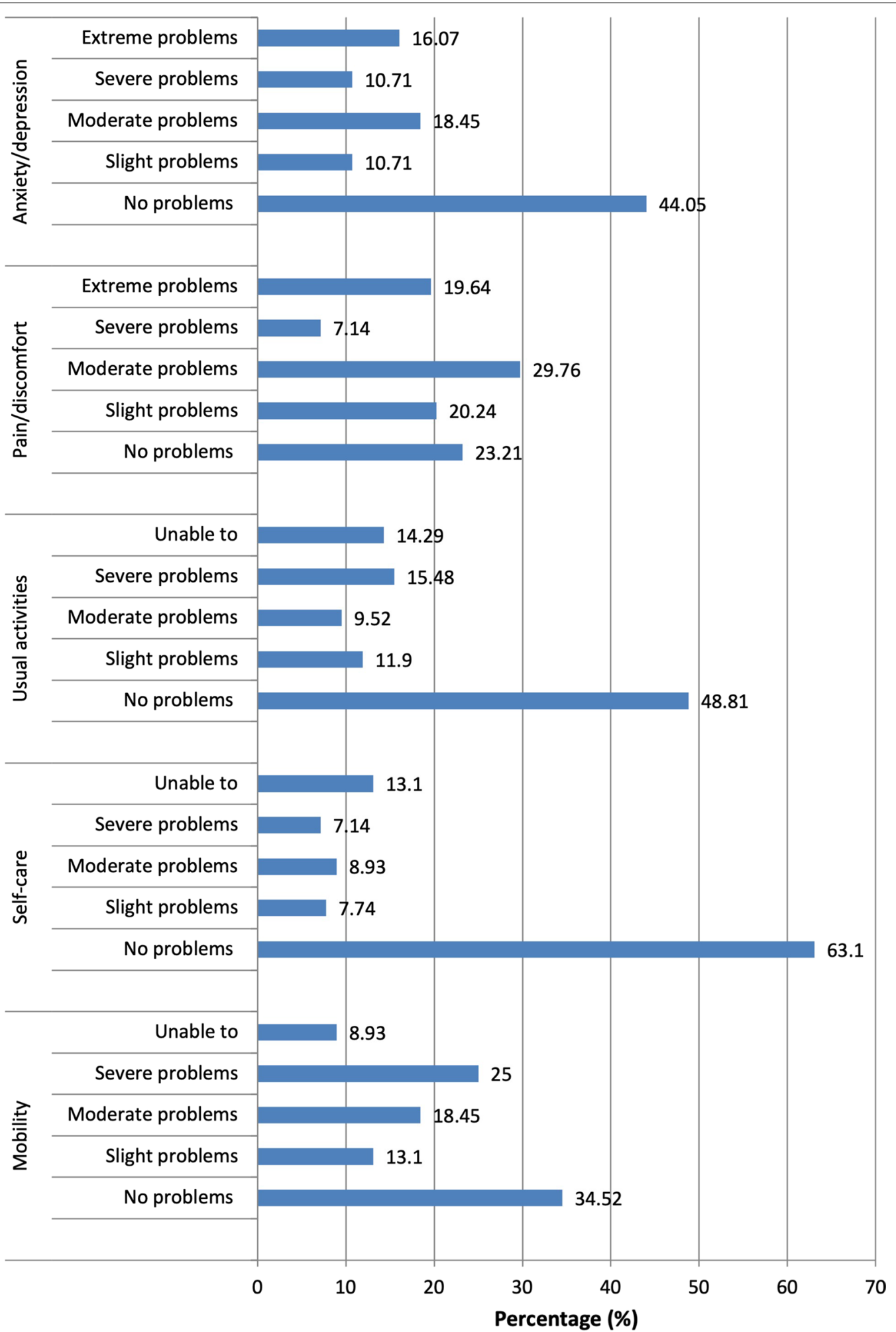

Fig. 1 The distribution of health-related quality of life measures using (EQ-5D-5L) 
Table 3 Predictors of quality of life among HD patients

\begin{tabular}{|c|c|c|c|}
\hline Variable & Beta & (Cl) $95 \%$ & $P$ value* \\
\hline Age & -0.0779 & $(-0.1549$ to -0.0085$)$ & 0.048 \\
\hline \multicolumn{4}{|l|}{ Income per month } \\
\hline$<250 \mathrm{JD}$ & Ref & Ref & Ref \\
\hline 250-500 JD & 0.0544 & $(-0.04115$ to 0.1499$)$ & 0.263 \\
\hline$>500 \mathrm{JD}$ & 0.1871 & $(-0.0411$ to 0.1499$)$ & 0.003 \\
\hline Exercise & 0.1571 & $(0.0538-0.2605)$ & 0.003 \\
\hline \multicolumn{4}{|l|}{ Years under dialysis } \\
\hline$<2$ & Ref & Ref & Ref \\
\hline $2-5$ & -0.1017 & $(-0.2071$ to 0.0037$)$ & 0.058 \\
\hline$>5$ & -0.1982 & $(-0.3043$ to -0.920$)$ & $<0.001$ \\
\hline Comorbidity index & -0.0259 & $(-0.0407$ to -0.0111$)$ & 0.001 \\
\hline $\begin{array}{l}\text { Average patients com- } \\
\text { plains }\end{array}$ & -0.3225 & $(-0.4060$ to 0.2389 & $<0.001$ \\
\hline \multicolumn{4}{|c|}{$\begin{array}{l}\text { Variable included in the model were selected using backward stepwise process } \\
\text { with } P<0.2 \text { to stay }\end{array}$} \\
\hline $\begin{array}{l}\text { *Statistical significance of } \\
P<0.05\end{array}$ & ariate re & ession analysis was set & 2-sided \\
\hline
\end{tabular}

exercise acts as a positive predictor of HRQoL is in agreement with Tsai et al. finding [37]. Physical activity levels were shown to be lower in dialysis patients compared to healthy sedentary controls [38]. Furthermore, exercise may improve the physical function as one of the dimensions of quality of life.

While Khatib et al. reported no association between household income and HRQoL [25], other studies demonestrated an improved HRQoL with higher income in terms of physical functioning, pain and social aspects $(11,39)$, which is in agreement with the current study finding. Possibly, patients who have higher income tend to have more leisure time and better social life than those who have low income.

Overall, the study findings provide important implications in identifying factors that could compromise HRQoL of HD patients. Targeting modifiable factors should be an essential component in any future appropriate interventions aiming at enhanced HRQoL of patients undergoing HD. Learning about such factors is important to improve patient care.

Our results provide insights into implementing procedures to improve health outcomes in dialysis patients. The current study highlights the needs of healthcare providers to develop and implement effective strategies for HD patients to help them improving their quality of life. For instance, early and continous assessment of QOL is critical. Healthcare professionals may establish teaching programs that provide patients with knowledge about the seriousness of the disease and the positive impacts of improved adherence. Also, development of support groups including patients and a multidisciplanary team of nephrologists, nurses, pharmacists and social workers would be necessary to improve treatment outcomes.

This study has many strengths. It is the first to assess HRQoL among HD patients with anemia in Jordan. The study utilized face-to-face interviews conducted by one clinical pharmacist which ensure consistently in data collection. Also, the current study explored various factors that may impact HRQoL such as socio-demographics, medical, laboratory, and psychiatric measures. However, study limitations should be noted. The cross-sectional design is descriptive and not intended to identify a cause-effect relationship between variables and outcomes. The recruited sample was only from four centers in Jordan which affects the generalizability of the study findings. Also, psychiatric information using HADS and some of iron indices data were not available for all subjects. Nevertheless, the current investigation has a unique conribution to the litertaure as it provides necessary baseline information about variables associated with poor HRQoL among anemic HD patients.

\section{Conclusions}

The study elucidated poor HRQoL among HD patients with anemia. Various range of dimensions of life were negatively affected among HD patients, highlighting the need of the healthcare providers to develop and implement potential strategies that would improve QOL of this patient population. Future research should be directed towards well-designed prospective interventional studies that explore the efficacy of such approaches in improving health outcomes among HD patients.

\section{Acknowledgements \\ Authors thank all participants of the study.}

\section{Authors' contributions}

OYA: conceptualization, data curation, funding acquisition, investigation, methodology, formal analysis, writing — review and editing. EAS: conceptualization, investigation, methodology, formal analysis, writing-review and editing. SMA: conceptualization, data curation, methodology, formal analysis, writing — review and editing. ASJ: investigation, methodology, writingreview and editing. RIF: investigation, methodology, writing-review and editing. All authors approved the final version of the manuscript and agree to be accountable for all aspects of the work. All authors read and approved the final manuscript.

\section{Funding}

This work was supported by the Deanship of Scientific Research at Jordan University of Science and Technology (Grant Number: 241/2017).

\section{Availability of data materials}

The data that support the findings of this study are available on request from the corresponding author. The data are not publicly available due to privacy or ethical restrictions. 


\section{Declarations}

\section{Ethics approval and consent to participation}

The study protocol was approved by the institutional review board committees at Jordan University of Science and Technology and The Ministry of Health (Reference Number: 29/107/2017). Written informed consent was obtained from each patient.

\section{Competing interests}

The author(s) declare no competing interests.

\section{Author details}

'Department of Clinical Pharmacy, Faculty of Pharmacy, Jordan University of Science and Technology, Irbid 22110, Jordan. ${ }^{2}$ Department of Internal Medicine, School of Medicine, The University of Jordan, Amman, Jordan.

\section{Received: 13 May 2021 Accepted: 6 December 2021}

\section{Published online: 24 December 2021}

\section{References}

1. Levey AS, DeJong PE, Coresh J, Nahas ME, Astor BC, Matsushita K, et al. The definition, classification, and prognosis of chronic kidney disease: a KDIGO controversies conference report. Kidney Int. 2011;80(1):17-28.

2. Beto J, Bhatt N, Gerbeling T, Patel C, Drayer D. Overview of the 2017 KDIGO CKD-MBD Update: practice implications for adult hemodialysis patients. J Renal Nutr Off J Counc Renal Nutr Natl Kidney Found. 2019;29(1):2-15.

3. Isakova T, Nickolas TL, Denburg M, Yarlagadda S, Weiner DE, Gutierrez OM, et al. KDOQI US Commentary on the 2017 KDIGO clinical practice guideline update for the diagnosis, evaluation, prevention, and treatment of chronic kidney disease-mineral and bone disorder (CKD-MBD). Am J Kidney Dis. 2017;70(6):737-51

4. Ngai M, Lin V, Wong HC, Vathsala A, How P. Vitamin D status and its association with mineral and bone disorder in a multi-ethnic chronic kidney disease population. Clin Nephrol. 2014;82(4):231-9.

5. Shettigar R, Samaranayaka A, Schollum JBW, Wyeth EH, Derrett S, Walker RJ. Predictors of health deterioration among older new zealanders undergoing dialysis: a three-year accelerated longitudinal cohort study. Can J Kidney Health Dis. 2021;8:20543581211022210.

6. Del Vecchio L, Locatelli F. Anemia in chronic kidney disease patients: treatment recommendations and emerging therapies. Expert Rev Hematol. 2014;7(4):495-506.

7. Stauffer ME, Fan T. Prevalence of anemia in chronic kidney disease in the United States. PLoS ONE. 2014:9(1):e84943.

8. Drueke TB, Massy ZA. Oral or intravenous iron for anemia correction in chronic kidney disease? Kidney Int. 2015:88(4):673-5.

9. Pei M, Aguiar R, Pagels AA, Heimburger O, Stenvinkel P, Barany P, et al. Health-related quality of life as predictor of mortality in end-stage renal disease patients: an observational study. BMC Nephrol. 2019;20(1):144.

10. Intas G, Rokana V, Stergiannis P, Chalari E, Anagnostopoulos F. Sleeping disorders and health-related quality of life in hemodialysis patients with chronic renal disease in Greece. Adv Exp Med Biol. 2020;1196:73-83.

11. Lemos CF, Rodrigues MP, Veiga JR. Family income is associated with quality of life in patients with chronic kidney disease in the pre-dialysis phase: a cross sectional study. Health Qual Life Outcomes. 2015;13:202.

12. Surendra NK, Abdul Manaf MR, Hooi LS, Bavanandan S, Mohamad Nor FS, Shah Firdaus Khan S, et al. Health related quality of life of dialysis patients in Malaysia: Haemodialysis versus continuous ambulatory peritoneal dialysis. BMC Nephrol. 2019;20(1):151.

13. Zyoud SH, Daraghmeh DN, Mezyed DO, Khdeir RL, Sawafta MN, Ayaseh $N A$, et al. Factors affecting quality of life in patients on haemodialysis: a cross-sectional study from Palestine. BMC Nephrol. 2016;17(1):44.

14. Eriksson D, Goldsmith D, Teitsson S, Jackson J, van Nooten F. Crosssectional survey in CKD patients across Europe describing the association between quality of life and anaemia. BMC Nephrol. 2016;17(1):97.

15. Freburger JK, Ellis AR, Wang L, Butler AM, Kshirsagar AV, Winkelmayer WC, et al. Comparative effectiveness of iron and erythropoiesis-stimulating agent dosing on health-related quality of life in patients receiving hemodialysis. Am J Kidney Dis. 2016;67(2):271-82.

16. Mapes DL, Lopes AA, Satayathum S, McCullough KP, Goodkin DA, Locatelli F, et al. Health-related quality of life as a predictor of mortality and hospitalization: the Dialysis Outcomes and Practice Patterns Study (DOPPS). Kidney Int. 2003;64(1):339-49.

17. Cangini G, Rusolo D, Cappuccilli M, Donati G, La Manna G. Evolution of the concept of quality of life in the population in end stage renal disease: a systematic review of the literature. Clin Ter. 2019:170(4):e301-20.

18. Nabolsi MM, Wardam L, Al-Halabi JO. Quality of life, depression, adherence to treatment and illness perception of patients on haemodialysis. Int J Nurs Pract. 2015:21(1):1-10.

19. EuroQol Research Foundation. EQ-5D-5L|Valuation |Crosswalk Index Value Calculator. 2019. Available from: https://euroqol.org/eq-5d-instr uments/eq-5d-5l-about/valuation-standard-value-sets/crosswalk-indexvalue-calculator/. Accessed October 2021.

20. EuroQol Research Foundation. EQ-5D-5L User Guide, 2019. Available from: https://euroqol.org/publications/user-guides. Accessed October 2021

21. Aburuz S, Bulatova N, Twalbeh M, Gazawi M. The validity and reliability of the Arabic version of the EQ-5D: a study from Jordan. Ann Saudi Med 2009;29(4):304-8.

22. Bekairy AM, Bustami RT, Almotairi M, Jarab A, Katheri AM, Aldebasi TM et al. Validity and reliability of the Arabic version of the the EuroQOL (EQ5D): a study from Saudi Arabia. Int J Health Sci. 2018;12(2):16-20.

23. Jarab A, Alefishat E, Mukattash T, Albawab A, Abu-Farha R, McElnay J. Exploring variables associated with poor health-related quality of life in patients with type 2 diabetes in Jordan. J Pharml Health Serv Res. 2019:10(2):211-7.

24. Jarab AS, Almousa A, Rababa'h AM, Mukattash TL, Farha RA. Healthrelated quality of life and its associated factors among patients with angina in Jordan. Quality Life Res Int J Quality Asp Treat Care Rehabil. 2020;29(4):1027-35.

25. Khatib ST, Hemadneh MK, Hasan SA, Khazneh E, Zyoud SH. Quality of life in hemodialysis diabetic patients: a multicenter cross-sectional study from Palestine. BMC Nephrol. 2018;19(1):49.

26. Alshogran OY, Khalil AA, Oweis AO, Altawalbeh SM, Alqudah MAY. Association of brain-derived neurotrophic factor and interleukin-6 serum levels with depressive and anxiety symptoms in hemodialysis patients. Gen Hosp Psychiatry. 2018;53:25-31.

27. Terkawi AS, Tsang S, AlKahtani GJ, Al-Mousa SH, Al Musaed S, AlZoraigi US, et al. Development and validation of Arabic version of the Hospital Anxiety and Depression Scale. Saudi J Anaesth. 2017;11(Suppl 1):S11-8.

28. Hemmelgarn BR, Manns BJ, Quan H, Ghali WA. Adapting the Charlson Comorbidity Index for use in patients with ESRD. Am J Kidney Dis. 2003;42(1):125-32

29. Khalil AA, Darawad M, Al Gamal E, Hamdan-Mansour AM, Abed MAJ. Predictors of dietary and fluid non-adherence in Jordanian patients with end-stage renal disease receiving haemodialysis: a cross-sectional study. Clin Nurs. 2013;22(1-2):127-36.

30. Thaweethamcharoen T, Noparatayaporn P, Sritippayawan S, Aiyasanon N Comparison of EQ-5D-5L, VAS, and SF-6D in Thai patients on peritoneal dialysis. Value Health Reg Issues. 2019:18:59-64.

31. Nagasawa H, Tachi T, Sugita I, Esaki H, Yoshida A, Kanematsu Y, et al. The effect of quality of life on medication compliance among dialysis patients. Front Pharmacol. 2018;9:488

32. Kang GW, Lee IH, Ahn KS, Lee J, Ji Y, Woo J. Clinical and psychosocial factors predicting health-related quality of life in hemodialysis patients. Hemodial Int Sympos Home Hemodial. 2015;19(3):439-46.

33. Shdaifat EA, Latif JY, Razak BT, Abdul Manaf MR. Quality of Life of caregivers and patients undergoing haemodialysis at Ministry of Health, Jordan. Int J Appl Sci Technol. 2012:2(3):75-85.

34. Zyoud SH, Al-Jabi SW, Sweileh WM, Arandi DA, Dabeek SA, Esawi HH, et al. Relationship of treatment satisfaction to health-related quality of life among Palestinian patients with type 2 diabetes mellitus: Findings from a cross-sectional study. J Clin Transl Endocrinol. 2015:2(2):66-71.

35. Yang F, Griva K, Lau T, Vathsala A, Lee E, Ng HJ, et al. Health-related quality of life of Asian patients with end-stage renal disease (ESRD) in Singapore. Quality Res Int J Quality Asp Treat Care Rehabil. 2015;24(9):2163-71.

36. Lopes AA, Bragg-Gresham JL, Goodkin DA, Fukuhara S, Mapes DL, Young EW, et al. Factors associated with health-related quality of life among 
hemodialysis patients in the DOPPS. Quality Res Int J Quality Asp Treat Care Rehabil. 2007;16(4):545-57.

37. Tsai YC, Chen HM, Hsiao SM, Chen CS, Lin MY, Chiu YW, et al. Association of physical activity with cardiovascular and renal outcomes and quality of life in chronic kidney disease. PLoS ONE. 2017;12(8):e0183642.

38. Johansen KL, Chertow GM, Ng AV, Mulligan K, Carey S, Schoenfeld PY, et al. Physical activity levels in patients on hemodialysis and healthy sedentary controls. Kidney Int. 2000;57(6):2564-70.

39. Albatineh AN, Ibrahimou B. Factors associated with quality-of-life among Kuwaiti patients on maintenance hemodialysis. Psychol Health Med. 2019;24(8):1005-14.

\section{Publisher's Note}

Springer Nature remains neutral with regard to jurisdictional claims in published maps and institutional affiliations.

- fast, convenient online submission

- thorough peer review by experienced researchers in your field

- rapid publication on acceptance

- support for research data, including large and complex data types

- gold Open Access which fosters wider collaboration and increased citations

- maximum visibility for your research: over $100 \mathrm{M}$ website views per year

At $\mathrm{BMC}$, research is always in progress.

Learn more biomedcentral.com/submissions 